\title{
Post-polypectomy surveillance interval based on flexible spectral color imaging enhancement (FICE) with magnifying zoom imaging for optical biopsy
}

(1) $\odot \ominus$

\author{
Authors \\ Rodrigo de Rezende Zago, Pedro Popoutchi, Lucas Santana Nova da Costa, Marcelo Averbach
}

Institution

Hospital Sírio-Libanês, São Paulo, Brazil

submitted 3.1 .2018

accepted after revision 19.3.2018

Bibliography

DOI https://doi.org/10.1055/a-0629-8985 |

Endoscopy International Open 2018; 06: E1051-E1058

(c) Georg Thieme Verlag KG Stuttgart · New York

ISSN 2364-3722

Corresponding author

Rodrigo de Rezende Zago, Hospital Sírio-Libanês,

Endoscopy Center, Rua D. Adma Jafet, 115, Bela Vista, São

Paulo - SP, Brazil 01307-000

Fax: +55 113231-0800

rodrigorzago@gmail.com

\section{ABSTRACT}

Background and study aims Post-polypectomy surveillance interval (SI) is determined based on the number, size, and histology of colorectal polyps. Electronic chromoendoscopy in association with magnifying imaging colonoscopy allows "in vivo" polyp histology prediction. Colorectal polyps $\leq 5 \mathrm{~mm}$ can be resected and discarded without pathologic assessment if the endoscopic technology when used with high confidence provides $\geq 90 \%$ agreement between the post-polypectomy SI and the SI based on pathological assessment. The aim of this study was to evaluate

\section{Introduction}

Colorectal cancer (CRC) is one of the most common malignancies worldwide and is a major cause of death [1]. Colonoscopy has an important role in treating premalignant lesions and preventing progression to invasive cancer [2]. After a colonoscopy with polypectomy, patients are enrolled in a follow-up program, and colonoscopies are performed at suggested intervals. the agreement between the post-polypectomy SI based on flexible spectral color imaging enhancement (FICE) chromoendoscopy in association with magnified imaging and the pathology-based SI.

Patients and methods Each diagnosed colorectal polyp received a histology prediction (neoplastic or non-neoplastic) based on the FICE capillary-vessel pattern classification. Each prediction was classified as high or low confidence. SI based on the FICE prediction was compared to the pathology-based SI recommendation according to the US Multi-Society Task Force on Colorectal Cancer guideline. Sensitivity, specificity and accuracy of FICE in diagnosing neoplastic lesions were compared with the pathology assessment. Interobserver and intraobserver agreement for FICE-based SI predictions was evaluated using the kappa coefficient.

Results A total of 267 polyps had histology prediction assessed with high confidence in 136 patients. Sensitivity of FICE was $98.7 \%$ (95\% Cl: 93.5-99.3) and specificity was $62.5 \%$ (95\% Cl: 43.6-78.9). Prediction accuracy was $94.4 \%(95 \% \mathrm{Cl}: 88.6-96-1)$ in differentiating between neoplastic and non-neoplastic lesions. Therefore, magnifying FICE colonoscopy-based SI recommendation was consistent with pathological assessment in $88.3 \%$ of general cases (95\% Cl: $82.1-92.6)$ and in $89.7 \%(95 \% \mathrm{Cl}: 83-$ 94.5) of the high-confidence evaluation cases. The intraobserver agreement value for FICE-based SI predictions was 0.87 (high-confidence evaluations), and the interobserver agreement values were 0.78 (high- and low-confidence evaluations) and 0.82 (high-confidence evaluations) (95\% $\mathrm{Cl}$ : 0.79-0.95).

Conclusions FICE-based SI demonstrated $89.7 \%$ concordance with the pathology-based SI. 
carded without pathologic assessment due to the low risk of these lesions harboring high-grade dysplasia or invasive cancer [4]. The approach allows immediate determination of the SI for patients treated with polypectomy and can reduce costs related to pathological analysis of the polyps.

The flexible spectral imaging color enhancement (FICE) approach can be used with magnification and exhibits $98 \%$ accuracy in diagnosing neoplastic and non-neoplastic colonic lesions [5]. The American Society for Gastrointestinal Endoscopy (ASGE) Technology Committee proposed minimum acceptable performance thresholds for the "resect and discard" strategy. The thresholds were reported in the Preservation and Incorporation of Valuable endoscopic Innovations (PIVI) document on the real-time endoscopic histological assessment of small colorectal polyps. The document states colorectal polyps $\leq 5 \mathrm{~mm}$ can be resected and discarded without pathologic assessment. The endoscopic technology for determining the histology of polyps $\leq 5 \mathrm{~mm}$ when used with high confidence can be combined with histopathologic assessment of polyps $>5 \mathrm{~mm}$ and should provide $\geq 90 \%$ agreement regarding post-polypectomy surveillance interval (PPSI) assignments with decisions based on pathological assessment of all identified polyps. High-confidence prediction refers to the endoscopist's clinical judgment that a lesion has sufficient features to predict its histology. In cases of low-confidence prediction, the polyp should be resected and submitted for pathological assessment [4].

A recent review and meta-analysis for the PIVI stated that limited data are available and that further studies are needed to evaluate use of FICE in the "resect and discard strategy" [6]. The aim of this study was to evaluate concordance between post-polypectomy SI predictions based on FICE chromoendoscopy in association with magnifying imaging in clinical practice and pathology-based SI assessment.

\section{Patients and methods}

A cross-sectional study was performed. We considered eligible patients over 18 years of age who received a colonoscopy at the Sírio-Libanês Hospital (São Paulo, Brazil) for screening, CRC surveillance, or evaluation of abdominal pain or change in bowel habits. All patients signed informed consent for participation in the study.

The following patient exclusion criteria were used for this study: a personal or family history of polyposis, inflammatory bowel disease, previous colonic resection for CRC, incomplete colonoscopy, inadequate bowel preparation and a lack of recovered polyps.

Study inclusion criteria were presence of at least one polyp detected during colonoscopy, evaluated via digital chromoendoscopy (FICE) with magnification, and resected and sent for histopathological analysis.

All procedures were performed consecutively by colonoscopists who frequently use digital chromoendoscopy with FICE and had more than 5 years of relevant experience. The physicians perform an average of 1,000 colonoscopies per year. The bowel was prepared with $20 \%$ mannitol solution. We used highdefinition EC-590 ZW5 colonoscopes with a 4400 processor
(Fujinon Fujifilm Corporation, Saitama, Japan). Maximum magnification was 100-fold. Digital chromoendoscopy with FICE was performed with preset number 4 and wavelengths of $R$ $500 \mathrm{~nm}, \mathrm{G} 520 \mathrm{~nm}$, and B $405 \mathrm{~nm}$.

Polyps were initially evaluated with white light and then with digital chromoendoscopy (FICE) with magnification up to 100fold at the endoscopist's discretion. We used the classification described by Teixeira et al. [5], based on capillary-vessel pattern. In this classification, type I reflects a normal pattern of thin vessels with linear shapes and regular arrangement surrounding the mucosal crypts; type II reflects a pattern characterized by few vessels or marginal capillaries of thicker diameters that are uniform in shape and without dilatations. Polyps classified as type I or type II vessel patterns were considered non-neoplastic. Type III applies to polyps with numerous capillaries of thinner diameter that are irregular and tortuous, with point dilatations and periglandular arrangement. Type IV polyps have numerous long, spiral or straight vessels of thicker diameters, surrounding villous glands and type $\mathrm{V}$ polyps have pleomorphism of capillaries with abnormal arrangements and thick vessels with chaotic arrangements. Polyps with a type III, IV or V patterns were considered neoplastic lesions. In cases of suspected serrated sessile adenoma or traditional serrated adenoma, the polyps were classified as vascular pattern type II and were presumed to be neoplastic. The endoscopists classified the presumed histological diagnosis as high-or low-confidence based on the endoscopist's clinical judgment that the lesion had features enabling its pathological prediction.

After completing the "in vivo" histology evaluation, the polyps were resected using either the injection-assisted mucosectomy technique or the polypectomy technique. The polypectomy technique was performed using biopsy forceps or a polypectomy snare at the endoscopist's discretion. Resected polyps were formalin fixed and sent to the Pathology Department of Sírio-Libanês Hospital in different bottles. A histopathological evaluation of each specimen was performed with hematoxylin and eosin staining.

High-definition images of the polyp taken during the procedure were stored in a PACS-IMPAX system (Agfa HealthCare, Mortsel, Belgium) for subsequent interobserver and intraobserver agreement evaluation. SI was determined by the endoscopist based on his "in vivo" evaluation. Our main objective was to compare SI recommendation based on FICE evaluation with SI recommendations based on histological analysis, according to the Multi-Society Task Force ( $\triangleright$ Table 1 ). A secondary objective was to evaluate agreement among endoscopist regarding $\mathrm{SI}$ recommendations based on FICE. Evaluation of the intraobserver and interobserver agreement regarding $\mathrm{SI}$ determination with FICE was performed 3 months after colonoscopy using the stored high-definition images. All polyp images were reevaluated by the same endoscopist and by the other two other participating endoscopists. The recommended SI from the second evaluation was compared with the recommended SI from the first evaluation. Interobserver agreement was determined based on evaluation by the other two endoscopists. Intraobserver agreement was determined by comparing each endoscopists' first and second evaluations. Intraobser- 
- Table 1 SI classification according to colonoscopy findings and histological examination [3].

\begin{tabular}{|l|l|c|}
\hline Class & Finding & Interval \\
\hline 1 & Small hyperplastic polyps of the sigmoid and rectum & 10 years \\
\hline 2 & $1-2$ adenomas $<10 \mathrm{~mm}$ & $5-10$ years \\
\hline 3 & $3-10$ adenomas $<10 \mathrm{~mm}$ & 3 years \\
\hline 4 & More than 10 adenomas & $<3$ years \\
\hline 5 & Advanced adenoma $(\geq 10 \mathrm{~mm}$, villous or high grade) & 3 years \\
\hline 6 & Serrated polyp $<10 \mathrm{~mm}$ or without dysplasia & 5 years \\
\hline 7 & Serrated polyp $\geq 10 \mathrm{~mm}$ or with dysplasia or traditional serrated adenoma & 3 years \\
\hline
\end{tabular}

- Table 2 Cohen's kappa agreement rate interpretation.

\begin{tabular}{|l|l|}
\hline Value of K & Strength of agreement \\
\hline$<0.20$ & Poor \\
\hline $0.21-0.40$ & Fair \\
\hline $0.41-0.60$ & Moderate \\
\hline $0.61-0.80$ & Good \\
\hline $0.81-1.00$ & Very good \\
\hline
\end{tabular}

ver and interobserver agreement analysis was performed using Cohen's kappa coefficient ( $\triangleright$ Table 2 ).

Sample size was calculated based on a general agreement of $90 \%$ between the two methods with an alpha error of $5 \%$ and a test power of $90 \%$. Therefore, 136 patients with polyps were required for this study.

Published data [5] indicate that FICE exhibits $99 \%$ sensitivity and $95 \%$ specificity in differentiating between neoplastic and non-neoplastic lesions. Therefore, 126 patients with polyps were required for the study. Previously published colonoscopy quality indicators [7] suggest that $40 \%$ of patients present with at least one polyp. Therefore, identifying 136 patients with polyps required approximately 350 colonoscopies.

Three colonoscopists performed 384 consecutive colonoscopies from November 1, 2015 to March 1, 2016 ( $>$ Fig. 1 and - Table 3). A total of 295 polyps were identified in 163 patients and were classified as neoplastic or non-neoplastic, ( $>$ Table 4 and > Fig. 2, > Fig. 3, > Fig. 4, and > Fig.5), allowing measurement and comparison of FICE sensitivity, specificity, positive predictive value (PPV), negative predictive value (NPV) and diagnostic accuracy between FICE-based and pathology assessments.

All data were stored in a Microsoft Excel 2010 table and were analyzed with STATA 13 software. Agreement between the FICE-based SI and the histopathology-based SI was calculated with the $95 \%$ confidence interval $(\mathrm{Cl})$.

This study was approved by the research and ethical committee of Sírio-Libanês Hospital.

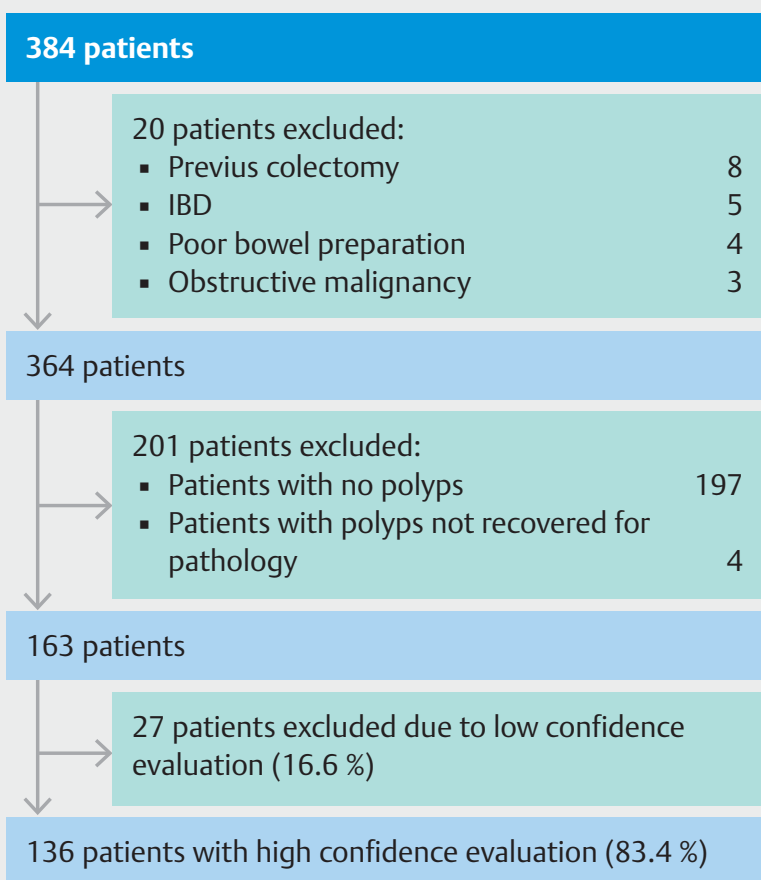

- Fig. 1 Patient selection process.

\section{Results}

FICE-based low- and high-confidence histology predictions and high-confidence predictions alone were compared with histopathological assessments and are shown in > Table 5 and $>$ Table 6. Performance of FICE in diagnosing neoplastic lesions "in vivo" with both high and low confidence and with high confidence only divided per segment is shown in > Table 7.

The general agreement between the FICE-based SI and histopathology-based SI recommendations was $88.3 \%(95 \% \mathrm{Cl}$ : $82.1-92.6 \%)$. The agreement was $89.7 \%$ (95\% Cl: $83-94.5 \%$ ) when only patients presumed to have high-confidence predictions were considered. Table 8 shows patients who were SI misclassified based on FICE evaluation. 
- Table 3 Patient characteristics.

\begin{tabular}{|l|c|c|}
\hline Patient & Number & Percentage (\%) \\
\hline Gender & & \\
\hline - Male & 84 & 51.5 \\
\hline - Female & 79 & 48.5 \\
\hline - Total & 163 & 100 \\
\hline Median age & $58 \pm 12$ & $25-82$ years \\
\hline Indication & & \\
\hline - Screening & 109 & 66. \\
\hline - Post-polypectomy Surveillance & 26 & 16 \\
\hline - Symptomatic & 28 & 17.8 \\
\hline Larger polyp size & & \\
\hline - $\leq 5$ mm & 83 & 50.9 \\
\hline - 6 - 9 mm & 51 & 31.2 \\
\hline - >10 mm & 29 & 17.7 \\
\hline Endoscopist & & \\
\hline - 1 & 24 & 17.1 \\
\hline - 2 & & 62.6 \\
\hline - 3 & 37 & 22.7 \\
\hline
\end{tabular}

FICE-based SI misclassified recommendations of all patients in this study showed that 11 (6.7\%) would have similar SI recommendations, 3 (1.8\%) would have delayed SI recommendations, and 4 (2.4\%) would have shortened SI recommendations. One patient would have the same interval recommendation by both methods. An examination restricted to patients with highconfidence FICE evaluations showed that 9 (6.6\%) would have similar SI recommendations, 2 (1.4\%) would have delayed SI recommendations, and 2 (1.4\%) would have shortened SI recommendations. One patient would have the same interval recommendation by both methods.

Interobserver agreement for the FICE-based SI determination was good (0.78) when both low- and high-confidence evaluations were considered. Agreement was very high (0.82) when only high-confidence presumptions were considered. General intraobserver agreement was also very good at 0.83 rate for high- and low-confidence evaluations and 0.87 for only high-confidence evaluations.

\section{Discussion}

Many endoscopy centers worldwide can diagnose polyp histology during a colonoscopy. Technologies based on enhancement of vascularization and evaluation of the pit pattern using magnification are commercially available. However, resecting all polyps and sending them for histopathological analysis remains the standard practice [6].
- Table 4 Polyp characteristics.

\begin{tabular}{|c|c|c|}
\hline Characteristic & \multicolumn{2}{|l|}{ Value } \\
\hline \multicolumn{3}{|l|}{ Size } \\
\hline - Median & \multicolumn{2}{|c|}{$5.6 \pm 3.9 \mathrm{~mm}$} \\
\hline - Variation & \multicolumn{2}{|c|}{$2-25 \mathrm{~mm}$} \\
\hline - Less than $5 \mathrm{~mm}$ & \multicolumn{2}{|c|}{$167(56.6 \%)$} \\
\hline - Between 5 and $9 \mathrm{~mm}$ & \multicolumn{2}{|c|}{$88(29.8 \%)$} \\
\hline - At least $10 \mathrm{~mm}$ & \multicolumn{2}{|c|}{$40(13.5 \%)$} \\
\hline Localization & Number & Percentage \\
\hline Cecum-ascending & 87 & 29.5 \\
\hline Transverse colon & 92 & 31.2 \\
\hline Descending colon & 33 & 11.2 \\
\hline Sigmoid and rectum & 83 & 28.1 \\
\hline - Total & 295 & 100 \\
\hline \multicolumn{3}{|l|}{ Paris classification } \\
\hline $.1 \mathrm{p}$ & 15 & 5 \\
\hline . Is & 203 & 68.8 \\
\hline - Ila & 62 & 21 \\
\hline - IIb & 15 & 4.8 \\
\hline \multicolumn{3}{|l|}{ Teixeira's classification } \\
\hline - Type 2 & 59 & 20 \\
\hline - Type 3 & 222 & 75.2 \\
\hline - Type 4 & 14 & 4.8 \\
\hline \multicolumn{3}{|c|}{ FICE-based presumptive diagnosis } \\
\hline - Neoplastic & 260 & 88.2 \\
\hline - Non-neoplastic & 35 & 11.8 \\
\hline \multicolumn{3}{|c|}{ High confidence FICE-based diagnosis } \\
\hline - Yes & 267 & 90.5 \\
\hline - No & 28 & 9.5 \\
\hline
\end{tabular}

Ignjatovic et al. [8] published the first study evaluating the clinical impact of the "resect and discard" strategy in 2009. Diagnoses were determined using narrow band imaging (NBI) and indigo carmine when necessary and were compared with histopathological analysis results. Sensitivity for detecting neoplastic polyps was $94 \%$ (95\% Cl: $90-97 \%)$. Specificity was $89 \%$ (95\% Cl: $78-95 \%$ ) and accuracy was $93 \%$ (95\% Cl: $89-96 \%)$. SI predictions were consistent with histopathology-based recommendations for 78 of 82 patients (95\%). The authors estimated that this strategy decreased costs by $77 \%$. Another published study [9] estimated that the "resect and discard" strategy for polyps smaller than $5 \mathrm{~mm}$ would eliminate up to $\$ 33$ million in healthcare costs per year in the United States.

Rex et al. [4] explored optical diagnosis of colorectal polyps. The data showed $90 \%$ agreement for SI determined by the 


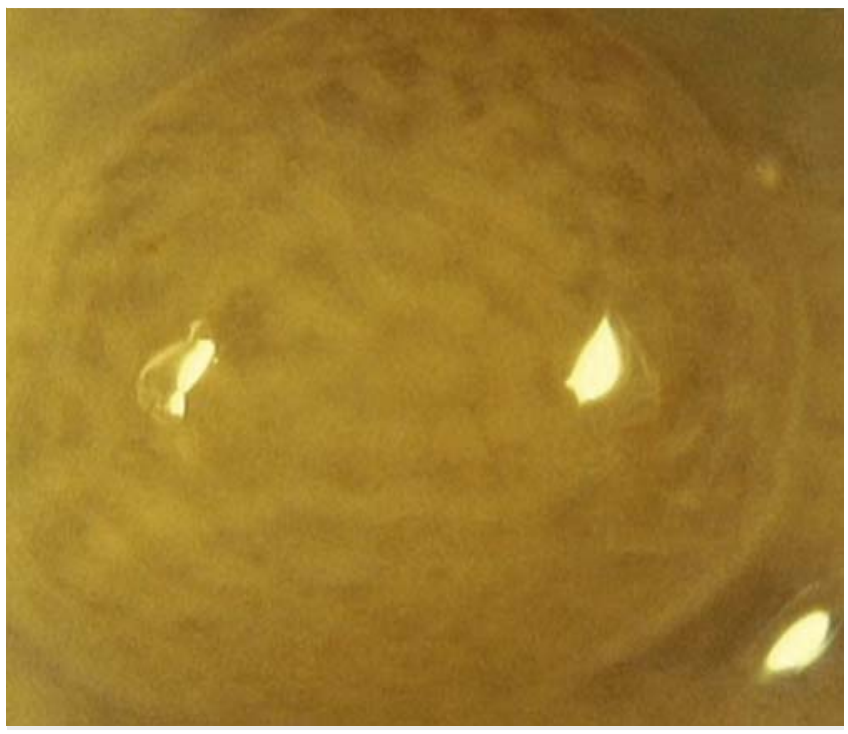

- Fig. 2 Polyp in the sigmoid colon evaluated with FICE and magnification. There are no identifiable capillaries (Teixeira's type class II). Pathology demonstrated a hyperplastic polyp.

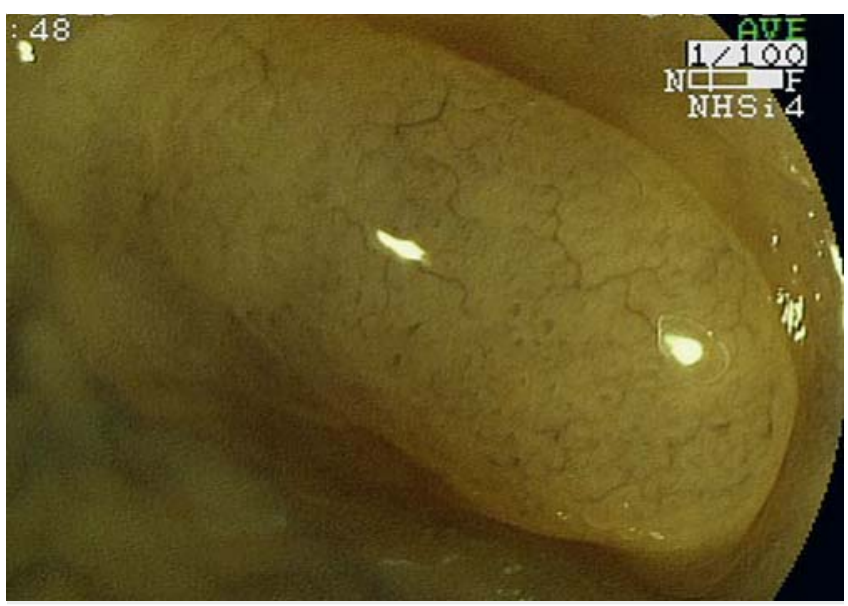

- Fig. 3 Polyp in the ascending colon evaluated with FICE and magnification. There are few marginal capillaries of thicker diameter, uniform and without dilatations (Teixeira's type II). This lesion was diagnosed as a sessile serrated adenoma.

method under evaluation using the "resect and discard" strategy for polyps smaller than $5 \mathrm{~mm}$.

Colonoscopies were performed by Teixeira et al. [5] using magnification and FICE to evaluate 309 colorectal lesions, which achieved $99.2 \%$ (95\% Cl: $98.2-100 \%$ ) sensitivity, $94.9 \%$ ( $95 \% \mathrm{Cl}: 92.5-97.4 \%$ ) specificity, and $98.3 \%$ global accuracy in differentiating neoplastic and non-neoplastic lesions. dos Santos et al. [10] used FICE to diagnose neoplastic and non-neoplastic lesions in 65 patients. The data showed the following results: $91.7 \%$ sensitivity, $95.7 \%$ specificity, $92.6 \%$ accuracy, $98 \%$ PPV and $78.6 \%$ NPV. Longcroft-Wheaton et al. $[11,12]$ also studied FICE for diagnosing neoplastic lesions in two studies; sensitivity, specificity, and accuracy were $88 \%$ to $93 \%, 81 \%$ to $82 \%$, and $86 \%$ to $89 \%$, respectively. Our results are similar to

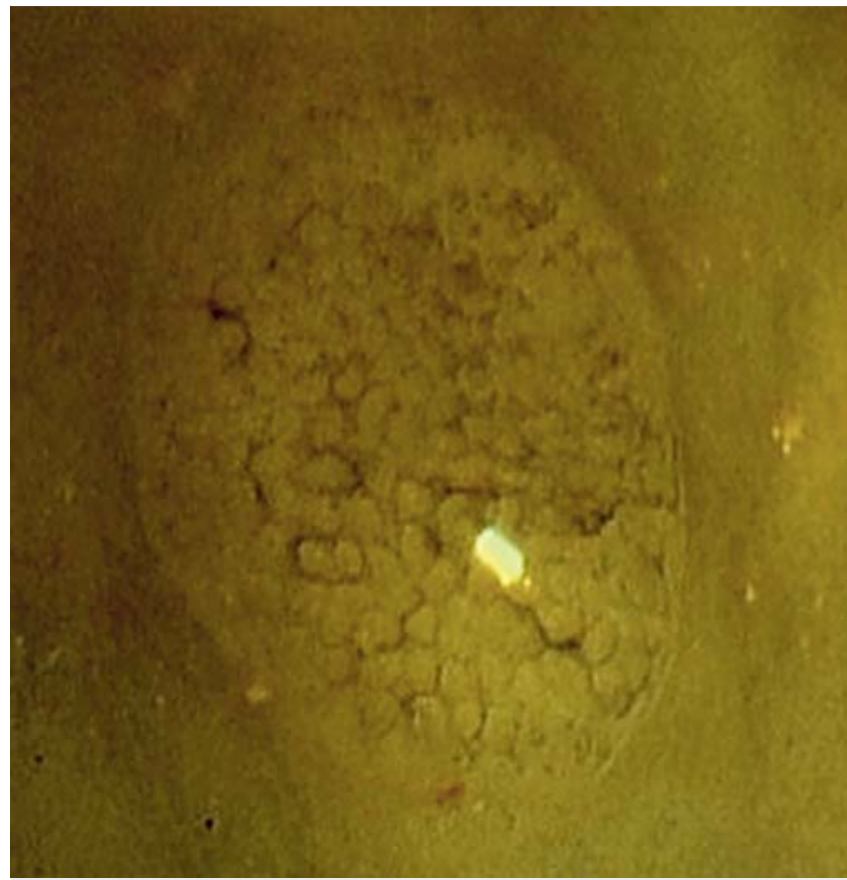

Fig. 4 Polyp evaluated with FICE chromoendoscopy and magnification. Note the numerous thinner capillaries with periglandular arrangement (Teixeira's type III), The resected specimen showed tubular adenoma histology.

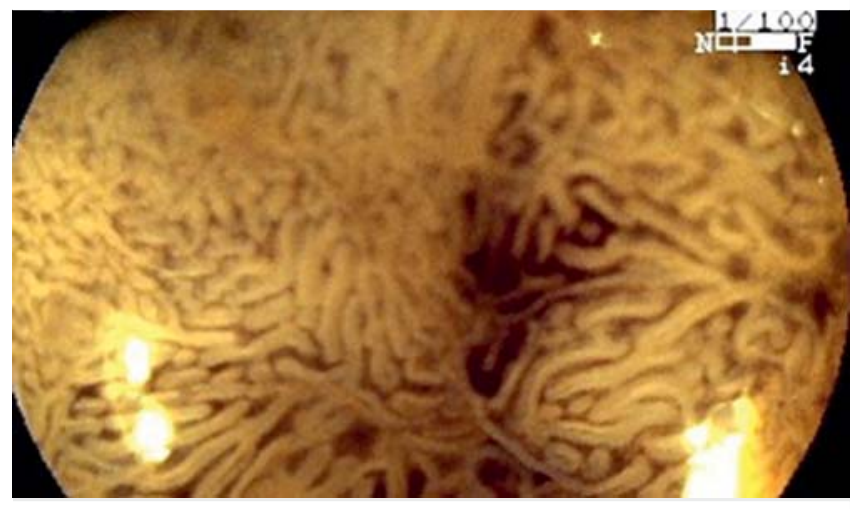

- Fig. 5 Polyp evaluated with FICE chromoendoscopy and magnification. Note the numerous long and spiral vessels of thicker diameter, surrounding villous glands (Teixeira's type IV). Pathology assessment confirmed tubulovillous adenoma.

the data published in the literature, even though we included lesions of all sizes. The original study by Teixeira et al. [5] evaluated lesions smaller than $10 \mathrm{~mm}$. The "resect and discard" strategy should be applied in clinical practice when minimum thresholds are met and only for lesions up to $5 \mathrm{~mm}$, but we analyzed performance of FICE in diagnosing neoplastic histology in lesions of all sizes, as this would reflect our daily practice.

In this study, we considered the endoscopist's confidence in polyp histology predictions. A high-confidence evaluation increased accuracy of lesion diagnosis by $2.2 \%$. Importantly, only $9.5 \%$ of lesions did not have characteristics allowing realtime diagnosis by the colonoscopist. The ASGE PIVI statement 
- Table 5 Number of polyps, FICE-based presumptive diagnosis (high and low confidence) and comparison with histology $(n=295)$.

\begin{tabular}{|l|l|l|r|}
\hline FICE & $\begin{array}{l}\text { Neoplastic } \\
\text { histology }\end{array}$ & $\begin{array}{l}\text { Non-neoplastic } \\
\text { histology }\end{array}$ & Total \\
\hline Neoplastic & 245 & 15 & 260 \\
\hline Non-neoplastic & 8 & 27 & 35 \\
\hline Total & 253 & 42 & 295 \\
\hline
\end{tabular}

- Table 6 Number of polyps, FICE-based diagnosis (high confidence) and comparison with histology $(n=267)$.

\begin{tabular}{|l|l|l|r|}
\hline FICE & $\begin{array}{l}\text { Neoplastic } \\
\text { histology }\end{array}$ & $\begin{array}{l}\text { Non-neoplastic } \\
\text { histology }\end{array}$ & Total \\
\hline Neoplastic & 232 & 12 & 244 \\
\hline Non-neoplastic & 3 & 20 & 23 \\
\hline Total & 235 & 32 & 267 \\
\hline
\end{tabular}

emphasizes the importance of clinical judgment in optical diagnosis. To our knowledge, few previously published studies analyzed this criterion.

FICE colonoscopies had lower specificity than previously published data and compared to that of other technologies such as NBI and Blue Laser Imaging (BLI), a recent technology released by Fujinon. In the last few years, NBI has been used in association with magnifying imaging. Kuruvilla et al. [13] used $\mathrm{NBI}$ in association with magnifying imaging for "in vivo" adenoma diagnosis and achieved $95 \%$ specificity, $97 \%$ NPV, $97 \%$ sensitivity, and 95\% PPV. Wallace et al. [14], used magnifying NBI and achieved $79 \%$ accuracy compared with pathology, with 88 $\%$ sensitivity and $66 \%$ specificity. Both studies used fixed-zoom magnification and the NICE classification for in vivo polyp histology assessment. NICE criteria evaluate the color, vessels and surface pattern of a lesion [15]. Yoshida et al. [16] adapted a classification system used with NBI to FICE chromoendoscopy, which assesses both surface patterns and capillary vessels. BLI is a relatively new technology, and few studies evaluating BLI imaging of surface pattern and capillary vessels have been published. Yoshida et al. [17] achieved 95.2\% accuracy in differentiating neoplastic and non-neoplastic polyps. When we developed this study, BLI was not available in our unit. In the near future, more studies can be designed to compare FICE and BLI chromoendoscopy, since the latest Fujinon equipment can perform both techniques.

Hyperplastic lesions have few vessels, which are generally regularly shaped in Teixeira's classification while non-neoplastic lesions commonly have no identifiable vessels in NBI examinations. Our low sensitivity may be partly explained by the low prevalence of non-neoplastic polyps in this study compared with that reported in the literature and use of a classification that considers only capillary vessel patterns and not surface patterns. Furthermore, sessile serrated lesions in the right co-

- Table 7 Sensitivity, specificity, positive predictive value (PPV), negative predictive value (NPV) and accuracy of FICE-based neoplastic polyps diagnosis compared with histology (general and per segment).

\begin{tabular}{|l|l|l|l|l|}
\hline Criteria & $\begin{array}{l}\text { General } \\
\text { (high and low confidence) }\end{array}$ & $\begin{array}{l}\text { General } \\
\text { (high confidence) }\end{array}$ & Right colon & Left colon \\
\hline Sensitivity & $96.8(93.8-98.6)$ & $98.7(96.3-99.7)$ & $97.5(93.5-99.3)$ & $95.6(89.1-98.7)$ \\
\hline Specificity & $64.2(48.0-78.4)$ & $62.5(43.6-78.9)$ & $52.9(27.8-77.0)$ & $72(50.6-87.9)$ \\
\hline PPV & $94.2(90.6-96.7)$ & $95.0(91.5-97.4)$ & $95.1(90.7-97.9)$ & $92.5(85.2-96.9)$ \\
\hline NPV & $77.1(59.8-89.5)$ & $86.9(66.4-97.2)$ & $69.2(38.5-90.9)$ & $81.8(59.7-94.8)$ \\
\hline Accuracy & $92.2(88.6-94.7)$ & $94.4(90.9-96.6)$ & $93.2(88.6-96.1)$ & $90.5(83.8-94.6)$ \\
\hline
\end{tabular}

- Table 8 Patients with incorrect SI assignments based on FICE evaluation (high and low confidence) ( $n=19)$.

\begin{tabular}{|l|l|l|l|l|}
\hline FICE-based SI class & Histology-based SI & $\begin{array}{l}\text { Number of patients } \\
\text { (high and low confidence) }\end{array}$ & $\begin{array}{l}\text { Number of patients } \\
\text { (high confidence) }\end{array}$ & $\begin{array}{l}\text { Outcome } \\
\text { (FICE } \mathbf{x} \text { histology) }\end{array}$ \\
\hline 1 & 2 & 4 & 3 & 10 years $\times 5-10$ years \\
\hline 2 & 1 & 7 & 6 & $5-10$ years $\times 10$ years \\
\hline 2 & 5 & 2 & 2 & $5-10$ years $\times 3$ years \\
\hline 3 & 2 & 4 & 2 & 3 years $\times 5-10$ years \\
\hline 5 & 7 & 1 & 1 & 3 years $\times 3$ years \\
\hline 6 & 7 & 1 & 0 & 5 years $\times 3$ years \\
\hline Total & & 19 & 14 & \\
\hline
\end{tabular}


Ion may appear to have a type II vessel pattern according to Teixeira's classification, leading to misdiagnosis. In addition, our study was not properly designed to evaluate the NPV in the context of the PIVI statement regarding the "do not resect and leave" strategy for non-neoplastic polyps, as we believe that the "resect and discard" strategy can have a greater clinical impact.

Post-polypectomy SI determination is based on polyp histology and size. However, visual estimation of polyp size is subject to errors. A recent study compared polyp size estimations by colonoscopists to resected specimen size for 1,528 lesions. Approximately $46 \%$ of the lesions were estimated to be larger than $10 \mathrm{~mm}$ by colonoscopists and $72 \%$ of these lesions corresponded to histopathological specimens smaller than $10 \mathrm{~mm}$ [18]. Chaptini et al. [19] studied polyp size evaluations by endoscopists using video images and assessed the impact of errors on SI recommendations. In $48 \%$ of cases $(95 \% \mathrm{Cl}: 39-$ $59 \%)$, polyp size was correctly estimated with a $20 \%$ margin of error; polyp size was overestimated in $32 \%$ of cases $(95 \% \mathrm{Cl}$ : $15-49 \%)$ and underestimated in $20 \%$ of cases $(95 \% \mathrm{Cl}: 4-$ $40 \%)$. Inaccurate visual estimation can lead to an inadequate SI recommendation for $10 \%$ of patients ( $95 \% \mathrm{Cl}$ : $5-14 \%)$. Several authors suggest the use of various tools as physical references to evaluate polyp size, such as biopsy forceps or modified accessories that can serve as a ruler [20]. We used visual estimation made by experienced colonoscopists, which is the most common procedure in daily practice. An evaluation of the intraobserver and interobserver agreement was performed to reduce this bias in our study.

FICE colonoscopy-based SI predictions showed 83.3\% (95\% $\mathrm{Cl}: 82.1-92.6 \%$ ) agreement with the histopathology-based $\mathrm{SI}$ recommendations according to the Guidelines for Colonoscopy Surveillance After Screening and Polypectomy: A Consensus Update by the US Multi-Society Task Force on Colorectal Cancer [21]. When we only evaluated the high-confidence presumptions, the agreement was $89.7 \%$ (95\% Cl: $83-94.5 \%)$. The confidence interval approaches the $90 \%$ agreement rate suggested by ASGE but we did not reach the minimum threshold for clinical practice [4]. A few patients were allocated in the wrong SI category but were assigned the correct SI by FICE examination. Although this inconsistency would result in appropriate followup in clinical practice, this should be regarded as the wrong classification in the research setting, as SI recommendations may change over time as new evidence emerges and our data may be analyzed retrospectively. In cases of disagreement regarding the SI, we found fewer than $2 \%$ of patients would be assigned a delayed SI using FICE, which could be associated with a higher risk of unfavorable outcomes, such as interval cancer.

Two previous studies by Longcroft-Wheaton et al. [11,12] evaluated the FICE-based interval predictions and found $97 \%$ agreement ( $\mathrm{Cl} 95 \%$ : $89-100 \%)$ in 67 patients and $100 \%$ agreement in 38 patients with the SIs determined by histopathological analysis. A meta-analysis was published recently on realtime polyp histology diagnosis by endoscopists. Evaluation of $\mathrm{NBI}$-based SI predictions included 10 studies with 3,082 patients. Agreement between the NBI-based SI and the histology-based SI recommendations was 89\% (Cl 95\%: 85-93\%).
Factors associated with greater than $90 \%$ SI agreement regarding the $\mathrm{SI}$ include studies performed in teaching hospitals, experienced colonoscopists, and high-confidence histological presumption. However, only two FICE-based SI publications were included in the meta-analysis [6]. Two more recent studies using NBI with magnification achieved agreement rates of $94 \%$ and $96 \%$ with pathology for the SI $[13,14]$.

Using the "resect and discard" strategy without histopathological analysis requires several considerations. A decision must be determined together with the patient to avoid potential ethical-legal concerns. In addition, high-quality images should be used. Published data show that $66.3 \%$ of 708 patients would accept the strategy in this study. Conversely, $48.9 \%$ of patients would accept this strategy only if the risk of cancer in very small polyps was zero. In addition, $62 \%$ would accept the costs for histopathological analysis [22].

Our evaluation of the intraobserver and interobserver agreement was performed to verify concordance in SI determination, which represents the next clinical decision after the in vivo polyp histology diagnosis. Very high agreement was observed for both interobserver evaluations with high confidence and in the intraobserver evaluations among the examiners. Previous studies have shown similar results $[10,21]$.

There are several limitations in this study. First, it was a single-center study. Although experienced endoscopists performed all of the procedures, no prior training was provided for standardization regarding FICE use. As a teaching unit with fellows, we may study learning curves of FICE and BLI among endoscopists in training who are unfamiliar with in vivo histology diagnosis in the future. More multicenter studies are needed, including studies in community centers, using the same wavelength proposed by Teixeira to enable reproducibility of the FICE classification for evaluating in vivo diagnostic performance before the "resect and discard" strategy can be incorporated into routine clinical practice.

\section{Conclusion}

In conclusion, we found that FICE-based post-polypectomy SI predictions showed high agreement with histology-based SI recommendations in this study, but this agreement was not sufficient for adoption of the "resect and discard" strategy. FICE chromoendoscopy showed high sensitivity and accuracy in diagnosing neoplastic polyps in the colon and rectum. Furthermore, intraobserver and interobserver agreement regarding FICE-based SI predictions was high.

\section{Competing interests}

None

\section{References}

[1] ACS - American Cancer Society (2016) Cancer facts \& figures. [cited 2016 Abr 3]. Available from: http://www.inca.gov.br/estimativa/2016 
[2] Winawer S], Zauber AG, Ho MN et al. Prevention of colorectal cancer by colonoscopic polypectomy. The National Polyp Study Workgroup. N Engl J Med 1993; 329: 1977 -1981

[3] Vieth M, Quirke P, Lambert R et al. Chapter 7 Annex: annotations of colorectal lesions. In: Segnan N, Patnick J, von Karsa L (editors). European guidelines for quality assurance in colorectal cancer screening and diagnosis. Luxembourg: European Union; 2010: 235-250

[4] Rex DK, Kahi C, O'Brien M et al. The American Society for Gastrointestinal Endoscopy PIVI (Preservation and Incorporation of Valuable Endoscopic Innovations) on real-time endoscopic assessment of the histology of diminutive colorectal polyps. Gastrointest Endosc 2011; 73: $419-422$

[5] Teixeira CR, Torresini RS, Canali C et al. Endoscopic classification of the capillary-vessel pattern of colorectal lesions by spectral estimation technology and magnifying zoom imaging. Gastrointest Endosc 2009; 69: 750 - 756

[6] Abu DayyehBK, Thosani N, Konda V et al. ASGE Technology Committee systematic review and meta-analysis assessing the ASGE PIVI thresholds for adopting real-time endoscopic assessment of the histology of diminutive colorectal polyps. Gastrointest Endosc 2015; 81: $502-516$

[7] Williams JE, Le TD, Faigel DO. Polypectomy rate as a quality measure for colonoscopy. Gastrointest Endosc 2011; 73: 498 - 506

[8] Ignjatovic A, East JE, Suzuki N et al. Optical diagnosis of small colorectal polyps at routine colonoscopy (Detect InSpect ChAracterise Resect and Discard; DISCARD trial): a prospective cohort study. Lancet Oncol 2009; 10: $1171-1178$

[9] Hassan C, Pickhardt PJ, Rex DK. A resect and discard strategy would improve cost-effectiveness of colorectal cancer screening. Clin Gastroenterol Hepatol 2010; 8: 865-869

[10] dos Santos CEO, Malaman D, Lopes CV et al. Digital chromoendoscopy for diagnosis of diminutive colorectal lesions. Diagn Ther Endosc 2012; 2012: 279521

[11] Longcroft-Wheaton GR, Higgins B, Bhandari P. Flexible spectral imaging color enhancement and indigo carmine in neoplasia diagnosis during colonoscopy: a large prospective UK series. Eur J Gastroenterol Hepatol 2011; 23: 903-911
[12] Longcroft-Wheaton G, Brown ], Cowlishaw D et al. High-definition vs. standard-definition colonoscopy in the characterization of small colonic polyps: results from a randomized trial. Endoscopy 2012; 44: 905-910

[13] Kuruvilla N, Paramsothy R, Gill R et al. A prospective dual-center proof-of-principle study evaluating the incremental benefit of narrow-band imaging with a fixed zoom function in real-time prediction of polyp histology. Can we resect and discard? Gastrointest Endosc 2015; 82: $362-369$

[14] Wallace MB, Crook JE, Coe S et al. Accuracy of in vivo colorectal polyp discrimination by using dual- focus high-definition narrow-band imaging colonoscopy. Gastrointest Endosc 2014; 80: 1072 - 1087

[15] Hewett DG, Kaltenbach T, Sano Y et al. Validation of a simple classification system for endoscopic diagnosis of small colorectal polyps using narrow-band imaging. Gastroenterology 2012; 143: 599-607

[16] Yoshida N, Naito Y, Kugai M et al. Efficacy of magnifying endoscopy with flexible spectral imaging color enhancement in the diagnosis of colorectal tumors. J Gastroenterol 2011; 46: 65 -72

[17] Yoshida N, Yagi N, Inada Y et al. Ability of a novel blue laser imaging system for the diagnosis of colorectal polyps. Dig Endosc 2014; 26: $250-258$

[18] Anderson BW, Smyrk TC, Anderson KS et al. Endoscopic overestimation of colorectal polyp size. Gastrointest Endosc 2016; 83: 201 - 208

[19] Chaptini L, Chaaya A, Depalma F et al. Variation in polyp size estimation among endoscopists and impact on surveillance intervals. Gastrointest Endosc 2014; 80: 652 - 659

[20] Kaz AM, Anwar A, O'Neill DR et al. Use of a novel polyp "ruler snare" improves estimation of colon polyp size. Gastrointest Endosc 2016; 83: $812-816$

[21] Lieberman DA, Rex DK, Winawer S] et al. Guidelines for colonoscopy surveillance after screening and polypectomy: a consensus update by the US Multi-Society Task Force on Colorectal Cancer. Gastroenterology 2012; 143: $844-857$

[22] Rex DK, Patel N], Vemulapalli KC. A survey of patient acceptance of resect and discard for diminutive polyps. Gastrointest Endosc 2015; 82: $376-380 . \mathrm{e} 1$ 